\title{
Narrativa minimista en la obra de Coriún Aharonián: análisis de la obra Pequeña pieza para piano I, de Coriún Aharonián
}

\author{
Luciana Orellana Lanús \\ Universidad Nacional de Cuyo. Facultad de \\ Artes y Diseño \\ lucianaorellanalanus@gmail.com
}

\begin{abstract}
Resumen: Nos proponemos a través de este escrito exponer diversos aspectos que se encuentren contemplados en la noción de "minimismo" latinoamericano. Para ello, es preciso definir que son Graciela Paraskevaídis (1940-2017), conjuntamente con Coriún Aharonián (1940-2017), los que intervienen en la enunciación de postulados acerca de ciertos rasgos en obras latinoamericanas. Desde esta perspectiva observamos que reparan no sólo en lo estrictamente musical, sino también en el contexto socio cultural, como asimismo en la toma de conciencia de una narratividad propia de la música latinoamericana contemporánea. Por tal motivo, pretendemos evidenciar a través del análisis de la primera de las Tres pequeñas piezas para piano (1966-1973), compuesta por Coriún Aharonián, ciertos materiales que den cuenta de producciones "minimistas". Conjuntamente con este análisis recuperaremos lo observado en la pieza ¿Y ahora?, del mismo autor, analizada con anterioridad.
\end{abstract}

Palabras clave: Minimismo latinoamericano, Coriún Aharonián, Música y sociedad

\section{Minimalist narrative in the work of Coriún Aharonián: analysis of the work Pequeña pieza para piano I, by Coriún Aharonián}

\begin{abstract}
We propose through this writing to expose various aspects that are contemplated in the notion of Latin American "minimalism". For this, it is necessary to define that Graciela Paraskevaídis (1940-2017), together with Coriún Aharonián (1940-2017), are those who intervene in the enunciation of postulates about certain features in Latin American works. From this perspective we observe that they pay attention not only to the strictly musical, but also to the socio-cultural context, as well as the awareness of a narrative characteristic of contemporary Latin American music. For this reason, we intend to show through the analysis of the first of the Tres pequeñas piezas para piano (Three Small Pieces for Piano, 1966-1973), composed by Coriún Aharonián, certain materials that account for "minimalist" productions. Together with this analysis, we will recover what was observed in the piece ¿Y ahora? (And now?) by the same author, analyzed previously.
\end{abstract}

Keywords: Latin American Minimism, Coriún Aharonián, Music and Society.

\section{Introducción}

En este artículo nos proponemos reflexionar sobre aspectos musicales que contribuyan a visibilizar ciertos rasgos contenidos en lo que Paraskevaídis y Aharonián

Este artículo desarrolla el trabajo presentado en el V Encontro Internacional de Teoria e Análise Musical, EITAM5 (LANÚS, 2019, p. 165-177). 
definen como "minimismo latinoamericano"1. Asimismo, consideramos que existen datos periféricos a estos postulados que implican una postura en la manera de producir música y de generar conocimiento específico sobre música latinoamericana contemporánea. Por esta razón, creemos que la disciplina del análisis musical es la que contribuye a dimensionar en las obras, no solamente lo estrictamente musical sino, además, una línea de pensamiento estético. En este sentido, partiremos de una breve referencia a la formación de estos compositores, conjuntamente con el pensamiento sobre música culta contemporánea, para luego concentrarnos en la enunciación de elementos que permitan esbozar una narratividad propia de ciertas músicas latinoamericanas. Una vez expuestas estas ideas, comenzaremos con el análisis musical de la primera de las Tres pequeñas piezas para piano de Coriún Aharonián. Por último, estableceremos un diálogo comparativo entre el análisis de la obra mencionada y la pieza ¿Y ahora? (1984), revisada en otra oportunidad.

Para comenzar, y específicamente respecto de la formación de los compositores, tanto Coriún Aharonián como Graciela Paraskevaídis tuvieron una estancia como becarios del Centro Latinoamericano de Altos Estudios Musicales (CLAEM). A raíz de la entrevista realizada en el libro de Hernán Vázquez (2015), inferimos que la permanencia de estos compositores en mencionado Centro resultó de suma importancia para su formación y vínculo con otros compositores latinoamericanos:

\begin{abstract}
Para mí había sido muy habitual buscar qué es lo que pasaba en Buenos Aires, y relacionarme con gente de mi generación, interesada con lo compositivo y con lo musicológico. O con mayores, como Juan Carlos Paz, como Hilda Dianda, como Fracisco Kröpfl. Ahí había estado estableciendo vínculos diversos, y en varios de esos vínculos aparecía la sombra del CLAEM. De una manera u otra iba apareciendo, e iba pesando poco a poco. [...] conocí a becarios de los bienios anteriores al mío con los que establecí vínculos, incluso de amistad, que perduran hasta ahora. (AHARONIÁN apud VÁZQUEZ, 2015, p. 34)
\end{abstract}

De la misma manera, Paraskevaídis manifiesta su paso por el CLAEM como una etapa determinante para la configuración de su estética:

\footnotetext{
${ }^{1}$ Este artículo propone ampliar o profundizar aquellos interrogantes que pueden haber surgido en relación a investigaciones anteriores.
} 


\begin{abstract}
En mi caso, cuando inicié la beca cumplía justo veinticinco años. En esa edad uno está abierto a todas las cosas, está ávido, y absorbe todo como una especie de esponja y luego eso va decantando, se va filtrando. Pero, debo decir [enfática] efectivamente mi pasaje por el CLAEM fue determinante para la creación musical que luego intenté desarrollar o llevar adelante. [...] A partir de ese momento tomé plena conciencia de mi responsabilidad $\mathrm{y}$, también, qué me interesaba hacer y qué quería o intentaba hacer. Eso se produjo, justamente, durante el período de beca porque confluyeron docentes, lecturas, materiales y conocimiento de otras obras. (PARASKEVAÍDIS apud VÁZQUEZ, 2015, p. 212)
\end{abstract}

Sumamos a este breve recorrido el relato de Aharonián en torno a la investigación de música culta en Uruguay, en donde también se menciona la relevancia del CLAEM:

\begin{abstract}
Varios uruguayos tuvieron vinculación con el Centro Latinoamericano de Altos Estudios Musicales que entre 1962 y 1971 dirigiera Alberto Ginastera dentro del Instituto Torcuato Di Tella en Buenos Aires. En primer término, el musicólogo Lauro Ayestarán, que fue convocado desde un comienzo como apoyo calificado, y que fue jurado del primer concurso de becas en 1962. En último término el compositor Héctor Tosar, que fuera invitado por Francisco Kröpfl en 1971 a cruzar el Plata regularmente a fin de iniciarse en las técnicas electroacústicas de composición. En el concurso bienal de jóvenes compositores latinoamericanos de 1968 fueron seleccionados Antonio Mastrogiovanni, Ariel Martínez y el autor de estas líneas. [...] El CLAEM se constituyó, en su escasa década de existencia, en un foco importantísimo de puesta al día y de confrontación de ideas para jóvenes compositores de distintos países de América Latina. (AHARONIÁN, 2013/2014, p. 62)
\end{abstract}

Remarcamos entonces la importancia de la participación y formación en el Instituto di Tella, así como la convergencia con otros compositores de Latinoamérica que luego significará para estos compositores un punto de encuentro con técnicas y pensamientos estéticos en torno a la identidad musical latinoamericana.

Por otro lado, es preciso mencionar la formación musical de Aharonián y Paraskevaídis con referentes de la música contemporánea tales como Lauro Ayestarán, Roberto García Morillo, Héctor Tosar, Gerardo Gandini, Francisco Kröpfl y Luigi Nono. Asimismo, ambos compositores resultan exponentes como gestores culturales, pedagogos y musicólogos, dejando un corpus de literatura sobre música latinoamericana y en particular sobre música culta contemporánea. Respecto de esto último, hemos de destacar 
una de las innovaciones y aportes significativos al campo de la música contemporánea: la conformación del Núcleo Música Nueva. Para Aharonián:

En esa época escribí mis primeros textos analizando el fenómeno de los Beatles, a fines de 1966. Ese año dimos comienzo a las actividades del Núcleo Música Nueva, con Ariel, con Conrado y con Daniel, con la colaboración de Mariano Etkin, Oscar Bazán, Luis Arias y Rafael AponteLedée, venidos de Buenos Aires, y con el apoyo tácito, desde allá, de Gerardo Gandini. [...] el Núcleo Música Nueva lleva 48 años de vida, también ininterrumpidos, difundiendo la música culta de vanguardia de todo el mundo, y especialmente la de América Latina y, por supuesto, la del Uruguay. Y se ha renovado generacionalmente varias veces, siempre basado en la labor militante. ¿Cuántas instituciones similares han sobrevivido medio siglo, y sin dinero? (AHARONIÁN apud CORRADO, 2014, p. 131, 142)

De igual modo, se puede establecer la ideología en la que se posiciona Aharonián de acuerdo a su incuestionable ética compositiva. Si bien es el entrevistador, Omar Corrado (2014) el que enumera estas características, Aharonián expresa estar de acuerdo con estas deducciones:

\begin{abstract}
En tus obras, así como en tus textos, se observa una constante preocupación por determinadas cuestiones que incluyen -centralmente, a mi juicio-, la necesidad de la contemporaneidad de los recursos, una lectura de la historia de la música en América Latina dirigida, entre otras cosas, a identificar constantes generadoras de tradiciones con las cuales dialogar creativamente en el propio trabajo compositivo, una actitud crítica hacia modelos y técnicas impuestos por distintos mecanismos y la posibilidad, en los mejores casos, de generar contramodelos. (AHARONIÁN apud CORRADO, 2014, p. 139-140)
\end{abstract}

Estos datos nos aportan información precisa sobre la concepción de su música en tanto que se cristaliza un posicionamiento anti colonialista, anti europeizante, que consideramos sumamente determinante para el concepto de "minimismo". En palabras de Tello:

\footnotetext{
Aharonián es un compositor con formación europea que se acerca miméticamente tanto de la música de la nativa población hace mucho tiempo diezmada como de la música popular uruguaya, la cual el conoce bien, a través de su contacto con músicos como arreglista. Su referencia a estas culturas musicales es ética: a través de su música él deja "hablar" otras voces culturales. Él conoce bien esas voces y las escucha en parte en sí
} 
mismo y en su entorno inmediato. Ellas son elaboradas en su práctica compositiva de manera que algunas de sus características sirven de base para sus ideas estructurales. (TELLO, 2008, p. 87)

Sumergido en estas preocupaciones, entendemos que es su actitud crítica ante el panorama de la música contemporánea la que imparte la diferenciación de obras "minimistas" en referencia a las obras minimalistas norteamericanas. A raíz de ello, en particular colocando el énfasis en la distinción terminológica que nos ocupa, es necesario abordar aquellas diferencias que plantean estos compositores y relacionar estos datos con la utilización de sus materiales musicales.

En primera instancia, llama nuestra atención que cuando Aharonián se refiere a los compositores como Terry Riley, Steve Reich y Philip Glass, lo hace en referencia a cuestiones estéticas. Para el autor, las obras de tales referentes del minimalismo norteamericano, reproducen 'fallas' estructurales “[...] se entusiasman con las posibilidades de la repetición, pero la entienden de otro modo y se instalan, de distinta manera, en una estilística adormecedora para el público y de mecanización maquinal para los intérpretes." (AHARONIÁN, 2002, p. 120). Afirmamos entonces, que, dado este posicionamiento, se impone una inquietud por recrear una perspectiva dialéctica propia respecto de músicas creadas por compositores latinoamericanos.

En segunda instancia, y en particular para abordar los rasgos que nos interesan en este escrito, damos cuenta de la necesidad del compositor de poder diferenciar aspectos constitutivos de un lenguaje en otro:

\footnotetext{
Por ejemplo, el minimalismo es en el norte principalmente un movimiento mecánicamente repetitivo, regresivo, neo reaccionario e, incluso, a menudo fascista. Mientras que, en el sur, las obras que podrían calificarse como minimalistas muestran un perfil que lucha contra la pasividad del oyente a través de una economía de medios, que multiplica lo expresivo potencial de recursos sólidos, de una estructuración reiterativa (no mecánica), de una preocupación por el timbre y la textura. Música inquietante en lugar de música para inducir el sueño. (AHARONIÁN, 2000, p. 5)
}

Advertimos a partir de esta expresión que la terminología empleada imparte un pensamiento en el que se evidencia la necesidad de emanciparse de prácticas incluidas en los territorios del "norte"; expresión irónica que acuñan ambos compositores: "El norte 
hegemónico nunca tuvo interés en abandonar su centro: mira, estudia, explica, juzga y domina el mundo desde allí. Nos explica cómo somos y nos indica qué y cómo hacer." (PARASKEVAÍDIS, 2014, p. 2).

Como venimos retratando, si bien se pone de manifiesto una postura política en estas apreciaciones, podemos afirmar que estas conjeturas nos resultan esclarecedoras en la medida que establecemos una diferenciación de los procesos norteamericanos aplicando la terminología adecuada a la narratividad latinoamericana:

\begin{abstract}
El manejo conceptual de estas premisas determina una esencialidad donde el silencio es parte de la estructura sonora y donde el minimismo (prefiero este término en castellano al de "minimalismo", el anglicismo en boga) resultante se opone al minimismo histórico estadounidense de la década de los sesenta, particularmente por la fuente y los objetivos que lo originan y sustentan: la observación y el rescate de ciertas prácticas musicales indígenas. (PARASKEVAÍDIS, 2014, p. 1)
\end{abstract}

De este modo, para concentrarnos específicamente en estas conjeturas, nos detendremos sobre una de las hipótesis que Aharonián expresa refiriéndose a la necesidad metodológica de abordar obras de autores latinoamericanos:

\begin{abstract}
Cualquier intento de teorizar sobre la composición latinoamericana necesariamente implicará un juicio previo sobre qué compositores considerar. Además, el contexto de un sistema colonial de transmisión cultural no se debe ignorar. Este contexto hace que sea importante evaluar la importancia de un compositor en términos de su carácter distintivo a la luz del compositor y de las composiciones metropolitanas. El número de tendencias características se puede observar en las obras de compositores que cumplen con estos criterios, incluido un sentido distintivo del tiempo; el uso de lo no discursivo, los procesos reiterativos; la austeridad; la violencia; la ruptura de las fronteras tecnológicas y culturales; y un interés en la identidad cultural. (AHARONIÁN, 2000, p. 1)
\end{abstract}

Siguiendo con esta línea de pensamiento, Paraskevaídis advierte, en particular con relación al análisis de la obra Austeras de Oscar Bazán (1936), información de suma relevancia para la construcción del concepto "minimismo latinoamericano":

En el V Curso Latinoamericano de Música Contemporánea (Buenos Aires, enero de 1976), el también cordobés Eduardo Bértola (1939-1996) se refirió a una música electroacústica "pobre", pero no en el sentido de arte povera, sino partiendo del desafío en América Latina de una voluntaria 
sobriedad y economía de medios, en el marco de una estética que opera exclusivamente con materiales esenciales, no retóricos y no discursivos. (PARASKEVAÍDIS, 2014, p. 2)

A propósito de ello, cabe mencionar como dato interesante que en el mismo curso de música contemporánea Oscar Bazán plantea el término “austeras" en concordancia con lo expuesto por Eduardo Bértola (1939-1996). Remarcamos así la significación de la obra a través de la interpretación de rasgos que den cuenta de una estética "pobre" o "austera".

Para concluir esta aproximación a mencionadas reflexiones estéticas, consideramos que, dadas estas especulaciones teóricas, la obra que logra reflejar estos aspectos es la primera de las piezas contenidas en Tres pequeñas piezas para piano, de Coriún Aharonián. Observando únicamente la partitura, podemos inferir a primera vista estos procesos de austeridad textural, de silencios expresivos, de reiteraciones no mecánicas y del placer por la violencia reprimida. Conjuntamente con la definición de los motivos, el sistema de organización de las alturas, las indicaciones de dinámica y otros parámetros, pensamos, dadas las características de esta música que la metodología de análisis de Francisco Kröpfl, sistematizada por María Inés García y María del Carmen Aguilar en Argentina, resulta adecuada para esta tarea. Por último, recuperaremos ciertos datos de ¿Y ahora? (1984), del mismo compositor, que nos permitan ejemplificar y contrastar con los rasgos que intentamos visibilizar en este escrito.

\section{Análisis de las obras}

Pequeña Pieza para Piano I forma parte del ciclo Tres pequeñas piezas para piano. Estas obras fueron compuestas en 1966 y 1973 y la primera de ellas resulta la más antigua. Asimismo, Aharonián decide colocarle un título sugerente a cada una de ellas. Así, las tres piezas poseen los dos títulos propuestos por el autor: un título sugerente y un título escrito en mayúscula (el que parece ser el más apropiado). El ciclo, en cuanto a los títulos, sería el siguiente:

- Pequeña pieza para gente que sufre la angustia en soledad o Pequeña pieza para piano $I$

- Pequeña pieza para gente con angustia colectiva o Pequeña pieza para piano II 
- Pequeña pieza para gente que superó la angustia o Pequeña pieza para piano III

Por otra parte, para abordar la obra, en primer lugar, nos proponemos reflexionar sobre los motivos ${ }^{2}$ que componen Pequeña pieza para gente que sufre la angustia en soledad o bien Pequeña pieza para piano I. Dada la recurrencia de los mismos, se produce una asociación inmediata a los procedimientos de compositores minimalistas como Steve Reich (1936) o Terry Riley (1935). De todas maneras, como hemos mencionado anteriormente, los rasgos distintivos propios y comunes a estos motivos poco tienen que ver con los que se exponen en las obras de Aharonián. De este modo, el primer motivo (Fig. 1) desde el punto de vista rítmico, posee un comienzo tético y un final resolutivo dado por el acento tónico y agógico (en el campo rítmico que plantea, la última figura es la más aguda y la más larga en cuanto a duración).

Figura 1 - Aharonián, Pequeña pieza para piano I, c. 1-2.

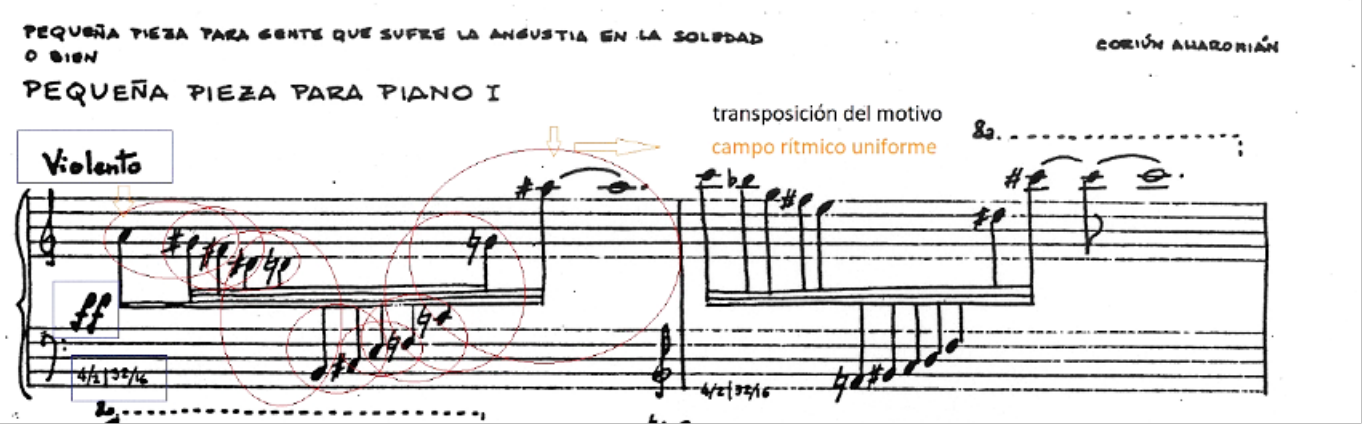

Fonte: Manuscrito de Aharonián.

Este motivo, en particular, utiliza un perfil melódico sumamente peculiar comparado al segundo material motívico que aparecerá de manera contrastante. El primero posee un gesto melódico del tipo escalístico a comparación con el segundo que está constituido como un bloque armónico.

\footnotetext{
${ }^{2}$ Un motivo para García, quien sistematiza la metodología de Francisco Kröpfl, es entendido como el conjunto mínimo con sentido musical, el cual genera elaboraciones.
} 
Del mismo modo, en este motivo y dada cierta predominancia del semitono, si lo analizamos en el campo de la música atonal observamos que utiliza tanto el micromodo ${ }^{3}$ menor como el micromodo mayor, lo que intuimos implica una sensación generalizada de atonalismo como así también de diatonismo.

Para una mejor comunicación de lo que estamos abordando con relación a la organización de las alturas, dejamos a continuación una clasificación (Fig. 2) de los micromodos de Francisco Kröpfl, sistematizados por María Inés García:

Figura 2 - Organización de las alturas.

1- Los que tienen como eje el semitono (micromodos menores)

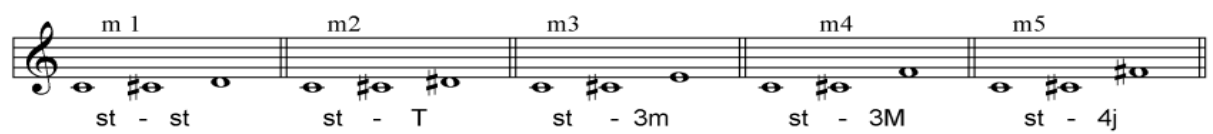

2- Los que tienen como eje la segunda mayor (micromodos mayores):

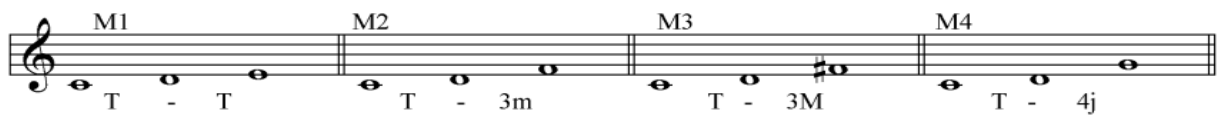

3- Los que tienen como eje la tercera menor:

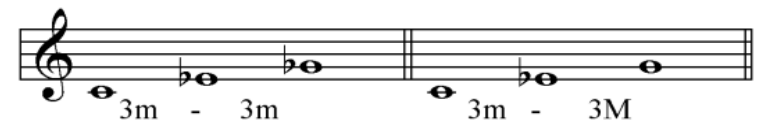

4- El que tiene dos terceras mayores:

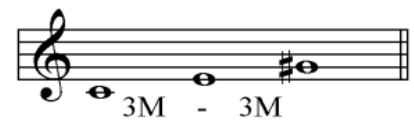

Fonte: García, Apuntes de cátedra, p. 2.

\footnotetext{
${ }^{3}$ El micromodo es el concepto propuesto en la metodología de Francisco Kröpfl para el análisis de las músicas atonales. Con una raíz en la "Teoría de los conjuntos" de Allen Forte, Kröpfl describe al micromodo como la red interválica más pequeña que puede darse en una permutación de sonidos. Así, en el total cromático, existirán doce permutaciones posibles. En oposición, el macromodo consiste en la sensación generalizada en donde predominan ciertas relaciones interválicas.
} 
En cuanto al metro, observamos que el autor describe una interpretación del motivo en torno a dos posibilidades. Por un lado, la indicación del 4/2 y por otro, la de 32/16. Inferimos que para Aharonián estas posibilidades métricas son referencias para el intérprete, de manera tal que logre dar cuenta de un campo rítmico no pulsado y la sensación de estímulo y movilidad. Así, en esta primera presentación del motivo, y en cuanto al aspecto rítmico, observamos una característica llamativa denominada "divergencias acentuales" presentes/detectadas también en los motivos de la pieza ¿Y ahora?, que en palabras de Kröpfl "generan procesos de tensión rítmica" (KRÖPFL, 1986, p. 14). Esta apreciación reconoce para nosotros un rasgo estético en el que podemos advertir una identidad en la narratividad musical de Aharonián:

[...] este es un aspecto del análisis musical al cual se le debe prestar especial y cuidadosa atención, pues, en gran medida, nos ayuda a revelar una serie importantísima de factores e interrelaciones estructurales que concurren a explicar por qué determinada obra musical presenta determinadas características distintivas que resultan fundamentales en cuanto a su realidad o identidad como forma sonora percibida. (GRELA, 1992, p. 27)

Por otro lado, yuxtapuesta a esta presentación se expone una transposición no exacta del motivo original. Estos cambios, minúsculos (tanto en las relaciones interválicas como en la primera figura del motivo), constituyen lo que en ocasiones anteriores hemos definido como desarrollo por microprocesos. Si recuperamos el análisis de la obra ${ }_{i} Y$ ahora?, el segundo motivo planteado en un campo rítmico uniforme al que considerábamos como el pulso de la obra (Fig. 3), también proponía leves modificaciones desde el lugar acentual en el que se presentaba (Fig. 4 y 5):

Figura 3 - Aharonián, ¿Y ahora?, c. 1-11.

motivo 1



Fonte: Manuscrito de Aharonián. 




Fonte: Manuscrito de Aharonián.

Figura 5 - Aharonián, ¿Y ahora?, c. 61-65.



Fonte: Manuscrito de Aharonián.

No obstante, un rasgo particular de estos motivos y que también advierte Herrera (2005), es que "la simplicidad y brevedad de los elementos utilizados es notable, así como el uso que Aharonián da a ellos" (HERRERA, 2005, p. 33). Es así que estos poseen características únicas (como personajes independientes) contrastantes en dinámicas, en textura, y en su densidad cronométrica. Asimismo, en ambas piezas todos los motivos son utilizados una cantidad limitada de veces, dato que inferimos a raíz de su distribución espacial en la partitura y en las indicaciones propuestas por el autor. 
Por otra parte, tal y como sucedía en ¿Y ahora?, Aharonián plantea una indicación diferente para cada uno de estos motivos (Cuadro 1):

Cuadro 1 - Cuadro comparativo.

\begin{tabular}{ll}
\hline ¿Y ahora? & Pequeña pieza para piano I \\
\hline $\begin{array}{l}\text { Primer motivo rítmico- melódico. Indicación: “como } \\
\text { con bronca, sin junar; non legato". La dinámica: } \\
\text { triple forte. }\end{array}$ & $\begin{array}{l}\text { Primer motivo rítmico- melódico. Indicación: } \\
\text { "violento". La dinámica: doble forte. }\end{array}$ \\
$\begin{array}{ll}\text { Segundo motivo rítmico-melódico. Indicación: } \\
\text { "calladito, non legato. La dinámica: quíntuple piano. }\end{array}$ & $\begin{array}{l}\text { Segundo motivo rítmico mélodico. En su } \\
\text { primera aparición no lleva indicaciones de } \\
\text { carácter. Dinámica: forte. }\end{array}$ \\
$\begin{array}{l}\text { Además, agrega: "pianísimo posible de acuerdo a la } \\
\text { variación démbrica inevitable, mano izquierda o }\end{array}$ & \\
derecha". & \\
$\begin{array}{l}\text { Tercer motivo rítmico-melódico. Indicación: } \\
\text { "puntiagudo, articulado". La dinámica: piano. }\end{array}$ & $\begin{array}{l}\text { Elaboración del segundo motivo rítmico- } \\
\text { melódico. Indicación: "Precipitato”. }\end{array}$ \\
$\begin{array}{l}\text { Cuarto motivo rítmico- melódico. Indicación: "gran } \\
\text { final, pero articulado". La dinámica es forte y agrega } \\
\text { sforzato en la primera figura y en la última. }\end{array}$ & $\begin{array}{l}\text { Recapitulación del motivo rímtico- melódico } \\
\text { uno (trasposición). Indicación: "dramático". }\end{array}$ \\
\end{tabular}

Fonte: Elaboración del autor, Orellana Lanús.

El segundo material motívico contrastante resulta sumamente ambiguo para el análisis (Fig. 6). Observamos que existe una célula que es la que caracteriza este segundo elemento. Sin embargo, el desarrollo completo de este material impone una reflexión en la que, desde el punto de vista de lo perceptivo, entendemos este discurso cuando finaliza su presentación (compás 6). Este rasgo le otorga una característica más temática ${ }^{4}$. Del mismo modo, la primera de estas células presenta un aspecto rítmico sencillo. Posee un comienzo tético y un final suspensivo. Esta célula rítmica que se repite, resulta el núcleo generador de este tema (si hemos de optar por clasificarlo de esta manera). A nivel de las alturas está constituido en un racimo de notas (clúster). Este conjunto de alturas en el comienzo plantea mayor diatonismo dada la predominancia de la segunda mayor como

\footnotetext{
${ }^{4}$ En la metodología de análisis abordada se imparte que el tema es una construcción rítmico melódica que caracteriza la obra y que, asimismo, es abarcable por la memoria inmediata del oyente. Esta teorización la realiza Kröpfl en torno a músicas tonales y funcionales. Sin embargo, dada la discursividad de este segmento, consideramos pertinente hablar de tema y célula.
} 
eje, convirtiéndose en un clúster más cromático como los señalados en celeste. Luego de un silencio, se articula una nueva aparición de la célula, que, tal y como venimos advirtiendo con el otro motivo, lo hace con una variante (tanto en las alturas como en el ritmo). Esta aparición, discursivamente, plantea el cierre de este pasaje lo que está en concordancia con la direccionalidad de este repertorio de alturas. Esto significa que, si observamos esta secuencia de alturas, entendemos que inicia en un registro agudo y finaliza en un registro más grave. Esta direccionalidad es la que también contribuye a considerar a este segmento como un bloque discursivo completo.

A propósito del metro, así como en la aparición del primer motivo, creemos que estos cambios métricos indicados por el compositor contribuyen a la presencia de un campo métrico no pulsado y a la percepción de un estímulo y posterior movilidad.

Figura 6 - Aharonián, Pequeña pieza para piano I, c. 4-6.



Fonte: Manuscrito de Aharonián.

A raíz de ello, podemos deducir también, que dadas las características de los motivos que estamos abordando desde la minuciosa descripción, se entiende un proceso de austeridad en tanto se presentan únicamente dos materiales que luego serán levemente modificados en el transcurso de la pieza: "El concepto de lo parco y austero abarca la utilización de pocos sonidos (en general y en particular) y se centra en un mínimo tensado hacia lo máximo [...] (PARASKEVAÍDIS, 2014, p. 1). Si bien estas conjeturas de Paraskevaídis se producen en torno a la obra Austera de Oscar Bazán, nos resulta esclarecedor dado que los elementos analizados en Pequeña pieza para piano I presentan rasgos similares. Destacamos entonces que, la economía de los materiales es una notoria característica en cierto repertorio de obras del compositor en cuestión. 
Por otro lado, no hablaremos en esta oportunidad de $\operatorname{sintaxis}^{5}$ dado que estas piezas estarían compuestas con los principios de sintaxis no discursiva, es decir, no se plantean en estas músicas las unidades sintácticas particulares de otras músicas y épocas.

A propósito de la separación y ahora refiriéndonos específicamente a la estratégica utilización del silencio, entendemos que en estas obras representan un discurso simbólico de suma trascendencia:

\begin{abstract}
En la música latinoamericana a partir de la década de 1970, aparecen varios aportes minimistas, cuyas características difieren, en origen y resultados, del minimismo estadounidense mencionado. La interacción sonido-silencio en la que el primero existe gracias al segundo y se constituye en un elemento estructural, y la fuerza motriz que mueve escasos elementos concentrados al máximo, se manifiestan a través de la utilización de secuencias de orden iterativo que imponen una presencia protagónica, gracias a sutiles variantes no mecánicas o previsiblemente mecanizadas. (PARASKEVAÍDIS, 1989, p. 2)
\end{abstract}

Observamos que el silencio se encuentra escrito de una manera tradicional, de modo tal que cuando realizan su entrada alguno de los motivos, el oyente se encuentre a la expectativa de los próximos eventos sonoros. Esto ocurre tanto en ¿YY ahora? como en Pequeña pieza para piano I (Fig. 7 y 8).

\begin{abstract}
En otras propuestas alternativas -tercermundistas o simplemente rioplatenses y no tan epigonales como pareciera a primera vista-, el objetivo es el opuesto: estructuras generativas breves, transformación interna que concentra la atención por lo imprevisible de su aparición, flexiones dinámicas, silencio expresivo y de tensión, ausencia de memoria histórica en lo armónico-tonal. (PARASKEVAÍDIS, 1989, p. 2)
\end{abstract}

\footnotetext{
${ }^{5}$ Una de las áreas propuestas por Francisco Kröpfl es la del análisis sintáctico o de las estructuras sintácticas
} 
Figura 7 - Aharonián, Pequeña pieza para piano I, c. 9-14.


Fonte: Manuscrito de Aharonián.

Figura 8 - Aharonián, Pequeña pieza para piano I, c. 15-18.

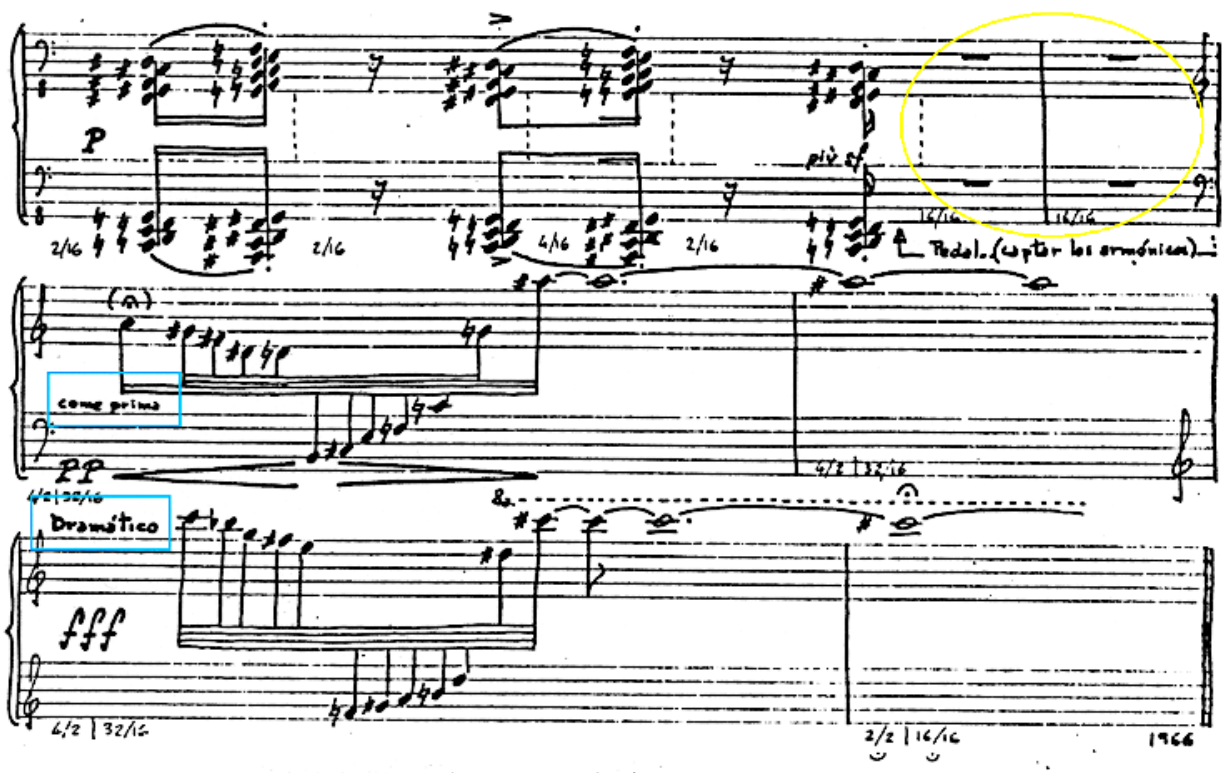

Fonte: Manuscrito de Aharonián.

Para finalizar, es necesario destacar los dos aspectos que nos ayudarán a reconstruir una posibilidad de análisis formal. En primer lugar, observaremos los procesos de densidad cronométrica y densidad textural en la obra ¿Y ahora? A partir del compás 25 , incluyendo los silencios dentro de esta unidad formal, podemos advertir que comienzan a estrecharse las apariciones de los motivos. Del mismo modo, es a partir del compás 25 
que estos se superponen por lo que se genera una mayor densidad textural sobre todo en el compás 56. La presentación del motivo cuatro delimita una última sección que posee un carácter más liquidativo. En este sentido, podemos determinar tres secciones. Un gráfico de estos aspectos resulta el siguiente ${ }^{6}$.

La primera sección (Tabla 1) está caracterizada por la presentación del motivo uno, dos y tres. La repentina aparición del uno, luego de la presentación del motivo dos, indicaría el final de un segmento.

Tabla 1 - Primera sección.

\begin{tabular}{|l|l|l|l|l|l|l|l|l|l|l|l|l|l|l|l|l|l|l|l|l|l|l|l|l|}
\hline & & & & & & & & & & & & & & & & $\mathrm{M} 3$ & & & & & & & & \\
\hline & $\mathrm{M} 1$ & & & & & & & & & & & & & & & & & & & & & & & $\mathrm{M} 1$ \\
\hline & & $\mathrm{M} 2$ & $\mathrm{M} 2$ & $\mathrm{M} 2$ & $\mathrm{M} 2$ & $\mathrm{M} 2$ & $\mathrm{M} 2$ & $\mathrm{M} 2$ & $\mathrm{M} 2$ & $\mathrm{M} 2$ & $\mathrm{M} 2$ & $\mathrm{M} 2$ & $\mathrm{M} 2$ & $\mathrm{M} 2$ & $\mathrm{M} 2$ & $\mathrm{M} 2$ & $\mathrm{M} 2$ & $\mathrm{M} 2$ & $\mathrm{M} 2$ & $\mathrm{M} 2$ & $\mathrm{M} 2$ & $\mathrm{M} 2$ & $\mathrm{M} 2$ & $\mathrm{M} 2$ \\
\hline & & & & & & & & & & & & & & & & & & & & & & & & \\
\hline 1 & 2 & 3 & 4 & 5 & 6 & 7 & 8 & 9 & 10 & 11 & 12 & 13 & 14 & 15 & 16 & 17 & 18 & 19 & 20 & 21 & 22 & 23 & 24 & 25 \\
\hline
\end{tabular}

Fonte: Elaboración del autor, Orellana Lanús.

Esta segunda sección está caracterizada por el aumento de los eventos sonoros en el tiempo y el aumento de la densidad textural. El silencio del compás 26 no solo genera expectativa, sino que favorece a la segmentación del discurso. Por estos motivos, lo ubicamos dentro del mismo.

Tabla 2 - Segunda sección.

\begin{tabular}{|l|l|l|l|l|l|l|l|l|l|l|l|l|l|l|l|l|l|}
\hline & & $\mathrm{M} 3$ & $\mathrm{M} 3$ & & & & & & $\mathrm{M} 3$ & $\mathrm{M} 3$ & & & $\mathrm{M} 3$ & & $\mathrm{M} 3$ & & \\
\hline & & & & & $\mathrm{M} 1$ & & & & $\mathrm{M} 1$ & & $\mathrm{M} 1$ & & & & & \\
\hline & & & & & & & $\mathrm{M} 2$ & $\mathrm{M} 2$ & $\mathrm{M} 2$ & $\mathrm{M} 2$ & $\mathrm{M} 2$ & $\mathrm{M} 2$ & $\mathrm{M} 2$ & $\mathrm{M} 2$ & $\mathrm{M} 2$ & \\
\hline & & & & & & & & & & & & & & & & & \\
\hline 26 & 27 \\
\hline 2 & 29 & 30 & 31 & 32 & 33 & 34 & 35 & 36 & 37 & 38 & 39 & 40 & 41 & 42 & 43 & 44 \\
\hline
\end{tabular}

Fonte: Elaboración del autor, Orellana Lanús.

\footnotetext{
${ }^{6}$ No se plantea una ubicación exacta y proporcional a las relaciones de alturas de los motivos rítmicomelódicos.
} 
Esta última sección se caracteriza por poseer el punto álgido, más significativo de la pieza (compás 56). Con apariciones más frecuentes de silencios y la "degradación" de los motivos, se plantea la finalización de esta estructura formal.

Tabla 3 - Tercera sección.

\begin{tabular}{|r|c|c|c|c|c|c|c|c|c|c|c|c|c|c|c|c|c||c|c|c|c|c|c|c|c|}
\hline & & & & & & & & & & & M3 & M3 & & & & & & & & & & & & M3 & \\
\hline & & & & & & & M1 & & & & & & & & & & & & M1 & & & & & & \\
\hline & & & & & & & & & M2 & M2 & M2 & M2 & & & M2 & M2 & & & & M2 & & & & & \\
\hline M4 & M4 & & & M4 & M4 & M4 & M4 & & & M4 & M4 & & & & & & & M4 & M4 & & & & & & \\
\hline 45 & 46 & 47 & 48 & 49 & 50 & 51 & 52 & 53 & 54 & 55 & 56 & 57 & 58 & 59 & 60 & 61 & 62 & 63 & 64 & 65 & 66 & 67 & 68 & 69 & 70 \\
\hline
\end{tabular}

Fonte: Elaboración del autor, Orellana Lanús.

Por otro lado, para analizar la forma de la Pequeña pieza para piano I, hemos de tener presente otros aspectos. Existe un principio de recurrencia esclarecedor en esta pieza. La aparición del primer material motívico (compás 17- figura 8), conjuntamente con la indicación del compositor "come prima", especifican una estructura ternaria. De todas maneras, el final de la primera de las secciones se entiende, así como en la pieza $¿ Y$ ahora?, por el contraste producido luego de una gran "pausa" (el silencio es estructural en estas obras). La aparición repentina de una nueva manifestación de la célula que caracterizaba el segundo elemento temático, contribuye a considerar la segunda sección de la pieza. Esta sección, asimismo, se distingue de la anterior incluso por la indicación del autor de cómo abordar el clúster (Fig. 9).

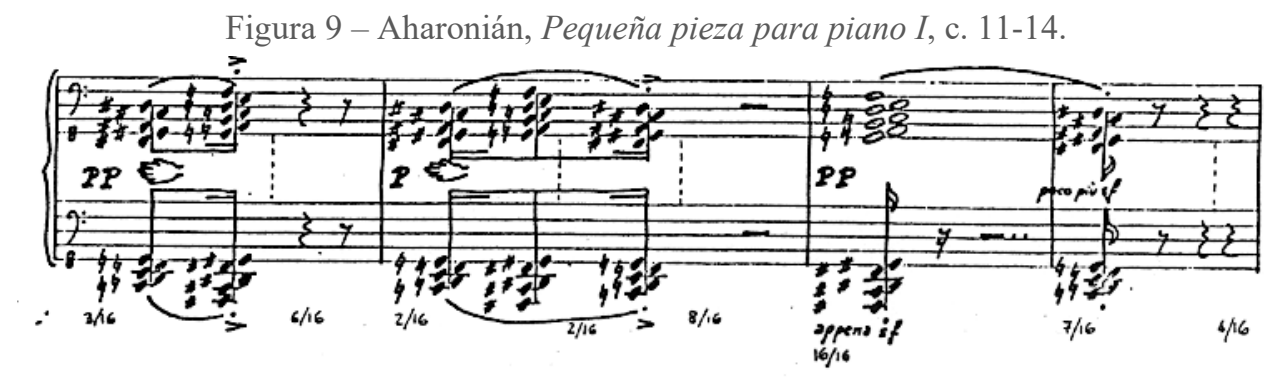

Fonte: Manuscrito de Aharonián. 
Por último, a diferencia del análisis formal de la obra ¿Y ahora?, no observamos en Pequeña pieza para piano I los procesos de superposición de los distintos materiales. Por lo tanto, el gráfico realizado para la obra anterior no resulta necesario en este caso dado que los principios generadores de la forma nos ayudan a reconstruir este discurso.

\section{Consideraciones finales}

Enunciadas las especulaciones teóricas en torno al concepto "minimismo" y establecidos los elementos de cada uno de estos análisis, resulta necesario formular una aproximación a la discursividad propia de Aharonián, como así también en torno a una narratividad propia de músicas latinoamericanas. Inferimos a la luz de estas particularidades observadas que existen mecanismos compositivos que implican una postura ideológica que se traduce en títulos sugerentes, en economía de materiales, en la utilización del silencio de una manera simbólica. De la misma manera, una aproximación a los rasgos rítmicos de los motivos, incluyendo las sutiles elaboraciones, nos otorgan la información acerca de lo que para estos compositores significa una música "pobre" o "austera". Asimismo, la repetición no mecánica de los materiales sonoros también parece ser un componente fundamental en esta categoría.

Tanto Aharonián como Paraskevaídis establecen una postura frente al panorama de la música contemporánea culta y esto se traduce en la apropiación de ciertos dispositivos que permiten diseñar una música "minimista". Este discurso impone la necesidad de una diferenciación con las músicas producidas por compositores norteamericanos como así también con otros discursos hegemónicos.

Es a través de este recorrido que comprendemos que el desarrollo por microprocesos, la dramática utilización del silencio, las indicaciones del tipo de carácter y la austeridad, son propias del texto musical de Coriún Aharonián. Damos cuenta, de esta manera, de obras que son coherentes en su formulación con un posicionamiento no solo político e ideológico, sino también estético. Este pensamiento estético es el que consideramos la punta para descubrir la identidad latinoamericana en músicas que propongan similares estrategias compositivas. En este sentido, la enunciación de estos postulados en torno al "minimismo" contribuyen a la creación de una metodología que 
consiste en la apropiación de discursos en donde la palabra identidad se pone de manifiesto.

Por último, estamos convencidos de que son necesarias nuevas líneas investigativas como así también análisis que permitan un acercamiento más exacto a estos procesos compositivos. Creemos que estas reflexiones son una deuda necesaria con la música actual latinoamericana.

\section{Referencias}

AGUILAR, María del Carmen. Formas en el tiempo: análisis musical para intérpretes. Buenos Aires: Edición de autor, 2015.

AHARONIÁN, Coriún. An Approach to Compositional Trends in Latin America. Leonardo Music Journal, v.10, p. 3-5, 2000.

AHARONIÁN, Coriún. Introducción a la música. Uruguay: Tacuabé, 2002.

AHARONIÁN, Coriún. La música uruguaya 1973-2013. In: OLIVERA, Rubén; AHARONIÁN, Coriún. Nuestro tiempo: Música. Libro de los bicentenarios, n. 5. Montevideo, Uruguay: Comisión del Bicentenario, 2013/2014. p. 5-63.

AHARONIÁN, Coriún. Pequeña pieza para piano I. [s.1.]: [s.n.], 1966. 1 partitura manuscrita cedida por Nairí Aharonián Paraskevaídis. Grabación disponible en: https://www.youtube.com/watch? $\mathrm{v}=$ jpijIL 7S4Wg\&ab_channel=musicnetmaterialsmusicnetmate rials. Acceso en: 23 mayo 2021.

AHARONIÁN, Coriún. ¿Y ahora?. [s.1.]: [s.n.], 1984. 1 partitura manuscrita cedida por Nairí Aharonián Paraskevaídis. Grabación disponible en: https://www.youtube.com/watch? $\mathrm{v}=\mathrm{x} 3 \mathrm{RVB} 9 \mathrm{~T}-$

PCc\&ab channel $=$ Cori $\% \mathrm{C} 3 \%$ BAnAharoni $\% \mathrm{C} 3 \% \mathrm{~A} 1 \mathrm{n}-$

TemaCori\%C3\%BAnAharoni\%C3\%A1n-Tema. Acceso en: 23 mayo 2021.

ARETZ, Isabel. América Latina en su música. México: Siglo XXI, 1985.

BÉHAGUE, Gerard. La música en América Latina: una introducción. Venezuela: Monte Ávila, 1983.

CORRADO, Omar. Coriún Aharonián: desde el Sur. Entrevista de Coriún Aharonián a Omar Corrado. El oído pensante, v. 2, n. 1, 2014. Disponible en: http://revistascientificas.filo.uba.ar/index.php/oidopensante/article/view/7447. Acceso en: 16 abr. 2021.

GARCÍA, María Inés. Metodología de análisis sintáctico-temática: apuntes de la Cátedra de Análisis y Morfología Musical. Facultad de Artes y Diseño, Universidad Nacional de Cuyo, 2011.

GRELA, Dante. La consideración de "las tendencias múltiples" (asociativas y disociativas) en el análisis musical. En serie 5: la música y el tiempo, n. 1, Rosario, Universidad Nacional de 
Rosario, p. 15-32, 1995.

GÓMEZ, Zoila et. al. Música latinoamericana y caribeña. Madrid: Pueblo y Educación, 1995.

HERRERA, Eduardo. Asuteridad, sintaxis no discursiva y microprocesos en la obra de Coriún Aharonián. Cuaderno de Música, Artes Visuales y Artes Escénicas, Colombia, v. 1, n. 1, p. 23$65,2005$.

KRÖPFL, Francisco. Propuesta para una metodología de análisis rítmico. In: SIMPOSIO DE ANÁLISIS MUSICAL, JORNADAS DE MUSICOLOGÍA, 3., 1986, Buenos Aires. Anais... Buenos Aires, Instituto Nacional de Musicología. Buenos Aires, 1986.

LANÚS, Luciana Orellana. En pos de una definición del "minimalismo latinoamericano". Análisis de la obra ¿Y Ahora?, de Coriún Aharonián. In: ENCONTRO INTERNACIONAL DE TEORIA Y ANÁLISE MUSICAL, 5., 2019, Campinas. Anais... Campinas, Universidade Estadual de Campinas, 2019, p. 179-189. Disponible en: https://eitam5.nics.unicamp.br/wpcontent/uploads/2020/12/EITAM5-paper_12_LanusL-pp_165-177.pdf. Acceso en: 27 mayo 2021.

MELLO, Chico. Coriún Aharonián y la mimesis a la constitución cultural latinoamericana. [s.l.], [s.n]. Versión en español, reducida y modificada, de las ultimas secciones del capítulo III de la tesis de doctoramiento. Disponible en: https://core.ac.uk/download/pdf/304709341.pdf. Acceso en: 20 sept. 2017.

MELLO, Chico. Mimesis und musikalische Konstruktion. Tesis (Doctorado en Musicología) Technischen Universität Dortmund, Dortmund, Alemania, 2008.

PARASKEVAÍDIS, Graciela. Las venas sonoras de la otra América. [s.1.]: [s.n], 2009. Versión corregida y ampliada de la Conferencia inaugural del simposio "La otra América", Escuela Superior de Música de Colonia, Alemania, 4-5 abr. 2009. Disponible en: http://www.gpmagma.net/pdf/txt e/sitio-lasvenassonoras.pdf . Acceso en: 31 ago. 2020.

PARASKEVAÍDIS, Graciela. Las austeras de Oscar Bazán. [s.1.]: [s.n], 2014. Disponible en: http://www.gp-magma.net/pdf/txt_e/sitio-Austeras.pdf. Acceso en: 20 ago. 2020.

PARASKEVAÍDIS, Graciela. El minimismo latinoamericano a través de la obra Piano piano del compositor uruguayo Carlos da Silveira. Pauta, Montevideo, n. 30, p. 74-83, 1989. Texto concebido en 1986. Disponible en: http://www.gp-magma.net/pdf/txt_e/gp-sitiopiano\%20piano.pdf. Acceso en: 20 ago. 2020.

VÁZQUEZ, Hernán Gabriel. Conversaciones en torno al CLAEM: Entrevistas a compositores becarios del Centro Latinoamericano de Altos Estudios Musicales. Buenos Aires: Instituto Nacional de Musicología "Carlos Vega", 2015. 